\title{
Fluid and Crystallized Intelligence and Broad Perceptual Factors Among 11 to 12 Year Olds
}

\author{
Lazar Stankov \\ University of Sydney, Sydney, Australia
}

\begin{abstract}
A battery of 36 visual and auditory tests was given to a sample of 113 primary school children. Second-order analysis of the data yielded two well-defined factors representing Fluid (Gf) and Crystallized (Gc) Intelligence and two perceptual factors corresponding to General Visualization (Gv) and General Auditory Function $(\mathrm{Ga})$. Perceptual factors were not clearly separated from broad intellective factors at this age level.
\end{abstract}

Factor analyses of large batteries of tests consisting of markers for the well-replicated primary abilities usually lead to extraction of two broad factors at the second order. Both factors involve the processes of perceiving relationships, educing correlates, reasoning, abstracting, attaining concepts, and solving problems, that is, the processes usually claimed to be important for intelligent behavior. One of these two factors, Fluid Intelligence (Gf), is involved in tasks in which relatively little advantage accrues from intensive or extended education and acculturation; the other one, Crystallized Intelligence $(\mathrm{Gc})$, represents tasks in which either the content or the operations involved depend on education and acculturation. This theory, now known as the theory of fluid and crystallized intelligence ( $\mathrm{Gf}-\mathrm{Gc}$ theory), was first proposed by Cattell and then supplemented by Horn (see Cattell, 1963, 1971; Horn, 1968, 1970, 1975; Horn \& Cattell, 1967).

Two features of the Gf-Gc theory that are relevant for the discussion here are (a) that it is a developmental theory of the structure of human abilities and (b) that it takes into

The work reported in this article was supported by the Institut za Pedagoška Istraživanja, Belgrade, Yugoslavia. Data analysis and write-up was done at the University of Sydney, Australia.

The author is grateful for helpful suggestions regarding the earlier draft of this article provided by J. A. Radcliffe. C. Latimer helped in correcting some of the more obvious language errors.

Requests for reprints should be sent to Lazar Stankov, Department of Psychology, University of Sidney, Sidney 2006, New South Wales, Australia. account the fact that, in addition to Gf and $\mathrm{Gc}$, there are several other broad factors operating within the putative measures of intelligence. With regard to the first feature, the references cited above provide the theoretical account and review of the relevant literature. Although Cattell (1967) reported data on children to check (at the subadult level) the course of development of Gf and Gc alone, most of the studies on Gf and Gc have been based on adult subjects.

Following Horn's formulations of the Gf-Gc theory, Undheim (1976) postulated that at the age of 10 to 11 years, when school has exerted influence for some years but little specialization has yet occurred, (a) the aspects of intelligence most tied to verbaleducation experience should be a tightly knit unit and (b) fluid and crystallized intelligence, if distinguishable at all, should be highly correlated, more so than has been observed in studies with adults. Undheim found evidence for both predictions. $\mathrm{He}$ also postulated and found evidence that at this age level, Gf and Gc could be distinguished from two other broad factors, that of Speediness (S) and General Visualization (Gv).

Similar hypotheses can be advanced regarding the age group on which the present study is based. The last-mentioned broad factor $(\mathrm{Gv})$ represents visual perceptual processes (such as imagining the way objects may change as they move in space, keeping configurations in mind, etc.) and reflects the fact that most ability tests use only one (visual) modality. Its repeated occurrence suggests that if the tests were devised to 
measure Gf and Gc through a different modality, then another perceptual factor would be necessary to reflect this modality. Stankov (1971) confirmed this hypothesis with auditory tests by obtaining a General Auditory Function (Ga) factor as well as Gf, Gc, and Gv. That study again used adults. The main concern here will be to discover whether the same four factors (i.e., Gf, Gc, $\mathrm{Gv}$, and $\mathrm{Ga}$ ) can be identified with younger subjects. Information on this question will contribute to knowledge of the developmental course of the perceptual factors and will also provide a check on what has been found regarding $\mathrm{Gf}$ and $\mathrm{Gc}$.

In addition to Horn's and Cattell's writings, a recent article by Bock (1973) gave a comprehensive summary of what is known. about Gv (though not stated in terms of Gf-Ge theory). Much less is known at present about the nature of $\mathrm{Ga}$. For that reason, before turning attention to the present study, it is necessary to review briefly some outcomes of the study in which Ga was discovered.

\section{General Auditory Function}

Stankov (1971) gave a battery of 71 tests to a sample $(N=241)$ of adult subjects aged 18 to 61 years. The battery consisted of two sets of tests. Those in the first set were visual tests measuring well-replicated primary abilities drawn mainly from the French, Ekstrom, and Price (1963) list and chosen to measure Gf, Gc, and Gv at the higher order. The second set of tests (50 altogether) consisted of auditory tests only, classifiable into the following categories: First, some tests were the same as the visual tests but were given through auditory channels (e.g., Vocabulary Test). Second, some tests were created by the author in order to be as close as possible to the visual tests. For example, an analogue of the Letter Series or Number Series Test would be an auditory test in which frequencies or intensities of tones were varied in such a way as to form a sequence. Third, some tests were standardized "musical abilities" tests, such as Seashore's and Wing's batteries, or were created on the basis of the descriptions of the tests used in speech perception studies (e.g., the White Noise Masking Test).
Factor analysis of the auditory tests alone produced seven factors (see those marked "auditory" in the modality column of Table 1). These seven factors, at the second order, formed three factors that were interpreted as Gf, Gc, and Ga. Extension analysis and the second-order analysis based on all 71 tests showed that Gf, Gc, Gv, and Ga could be clearly identified. Therefore, $\mathrm{Gf}$ and $\mathrm{Gc}$ can be tapped by both visual and auditory tests. Gv is defined by visual tests only, and it does overlap with Gf. Ga is defined by auditory tests, and it overlaps with Gc. Finally, there is a relatively small overlap between Gv and Ga. The General Auditory Function involved processes of recognizing words spoken with some kind of interference, maintaining steady tempo and recognizing changes in rhythmic patterns, keeping the order of presentation of tones in mind and recognizing changes in this order, and so on.

General Auditory Function correlated with age and with auditory loss detected by an audiometer test. This can be interpreted to mean that the decline in Ga during adult years depends on the reduction of auditory acuity commonly observed in older people. All this information was taken to indicate that $\mathrm{Ga}$ indeed represents broad auditory perceptual processes akin to Gv in the visual domain. Horn (1974) provides a detailed discussion of auditory factors and of their relationship with visual primaries.

\section{The Problem}

The aim of the present research is to investigate whether the results obtained by Stankov (1971) could be replicated with younger subjects and in a different culture. To this end, a battery of 36 tests was assembled to measure seven auditory factors, plus six visual primaries known to load Gf, $\mathrm{Gc}$, and Gv. An attempt was made to ensure that at least three tests defined every primary, but that was not possible due to the time limits imposed. Table 1 presents the list of primaries, variables used to assess these primaries, and hypothesized secondorder structure based on the previous studies.

Most of the primaries used here are the 
Table 1

Primary Factors, Tests Used to Assess Them, and Hypothesized Second-order Structure

\begin{tabular}{|c|c|c|c|c|c|c|c|}
\hline Primary factor & Symbol & Modality & Testa & $\mathrm{Gc}$ & Gf & Gv & $\mathrm{Ga}$ \\
\hline Verbal Comprehension & V & $\begin{array}{l}\text { Auditory } \\
+ \text { Visual }\end{array}$ & $1,2,3$ & $\mathrm{X}$ & & & \\
\hline Experiential Evaluation & EMS & Visual & $4,5,6$ & $\mathrm{X}$ & & & \\
\hline Induction & I & Visual & 7,8 & & $\mathrm{X}$ & & \\
\hline Auditory Induction & Ia & Auditory & $9,10,11$ & & $\mathrm{X}$ & & \\
\hline Memory Span & Ms & Auditory & $12,13,14$ & & $\mathrm{X}$ & & \\
\hline Temporal Reordering & $\operatorname{Tr}$ & Auditory & 15,16 & $\mathrm{X}$ & $\mathrm{X}$ & & \\
\hline Sound Pattern Recognition & $\operatorname{Pr}$ & Auditory & $17,18,19$ & $\mathrm{X}$ & $\mathrm{X}$ & & $\mathrm{X}$ \\
\hline Relation Perception & $\mathrm{Rp}$ & Auditory & 20,21 & & $\mathrm{X}$ & & $\mathrm{X}$ \\
\hline Spatial Scanning & Ss & Visual & $22,23,24$ & & & $\mathrm{X}$ & \\
\hline Flexibility of Closure & $\mathrm{Cf}$ & Visual & $25,26,27$ & & & $\mathrm{X}$ & \\
\hline Perceptual Speed & $\mathrm{P}$ & Visual & $28,29,30$ & & & $\mathrm{X}$ & \\
\hline Masking & $\mathrm{Mg}$ & Auditory & $31,32,33$ & & & & $\mathrm{x}$ \\
\hline Tempo & $\mathrm{T}$ & Auditory & $34,35,36$ & & & & $\mathrm{X}$ \\
\hline
\end{tabular}

Note. Gc represents the factor of Crystallized Intelligence; Gf, the factor of Fluid Intelligence; Gv, the factor of General Visualization; and $\mathrm{Ga}$, the General Auditory Function.

The test numbers correspond to the tests listed in Table 2.

same as those employed by Stankov (1971). There are two new primaries (Spatial Scanning and Perceptual Speed) that were not used before with auditory tests but are known to measure Gv. Also, two new tests were added as markers for Experiential Evaluation.

The auditory battery was slightly different from the previous study. All the tests used do represent markers for a particular primary. Every primary, however, was defined before by more than two or three variables, and the choice of particular markers here would have to change somewhat the nature of the primary itself. For example, Auditory Induction (Ia) is here defined by two tests that previously loaded on Reasoning $(R)$. In the previous study, Reasoning clearly involved both Inductive and Deductive Reasoning, but the markers chosen for this study were only those for Inductive Reasoning. Similarly, Tempo (T) here was a part of the Rhythm factor previously, and Relation Perception was previously a part of the Memory-Span Relation Perception factor.

Most of the visual primaries are well known and need not be discussed here. The auditory primaries are given operational definition by their marker tests. Temporal Reordering represents the ability to keep in mind the order of presentation of auditory stimuli and to recognize these stimuli when presented in a different order. Sound Pat- tern Recognition represents the ability to recognize a pattern consisting of series of either tones, chords, or letters. Relation Perception is rather heavily loaded with auditory memory, but its basic feature is that it involves the ability to perceive the relationship among auditory stimuli consisting of either tones or meaningful sentences. Masking is the ability to hear words spoken under conditions of various kinds of interference, and it can be interpreted as a figure-ground phenomenon. Finally, Tempo requires the ability to maintain steady tempo either during the silent interval or when an interfering beat exists. It is important in perceiving rhythm either of a musical kind or as it exists in spoken language.

The hypothesized loadings of the primaries on the second-order factors are also indicated in Table 1. It is this pattern that is explored here.

\section{Method}

\section{Subjects}

Subjects $(N=113)$ were fifth- and sixth-grade pupils from a primary school in a relatively homogeneous working-class suburb of Belgrade, Yugoslavia. Their age was 11 to 12 years at the time of testing in the spring of 1973 . About $50 \%$ were girls. Their native language was Serbo-Croatian, a dominant language of Yugoslavia. 
Table 2

Test Statistics

\begin{tabular}{|c|c|c|c|c|}
\hline Test & $\begin{array}{c}\text { No. } \\
\text { items }\end{array}$ & $M$ & $S D$ & $r_{x x}$ \\
\hline 1. Multiple-Choice Intelligibility & 60 & 44.38 & 9.94 & .80 \\
\hline 2. Cloze & 36 & 17.11 & 6.33 & $.63^{*}$ \\
\hline 3. Verbal Meaning & 24 & 15.49 & 5.23 & .75 \\
\hline 4. Social Situations & 14 & 6.36 & 1.80 & $.60^{*}$ \\
\hline 5. Perception of Human Behavior & 25 & 13.96 & 3.48 & .52 \\
\hline 6. Recognition of Emotions & 16 & 8.82 & 2.25 & $.65^{*}$ \\
\hline 7. Letter Series & 20 & 5.51 & 3.76 & .73 \\
\hline 8. Number Series & 20 & 7.98 & 4.37 & .58 \\
\hline 9. Sequential Tonal Series & 20 & 8.05 & 4.81 & .58 \\
\hline 10. Chord Series & 19 & 7.97 & 6.37 & .75 \\
\hline 11. Tonal "Gottschaldt" Figures & 20 & 9.22 & 6.31 & .84 \\
\hline 12. Number Span (Forward) & 12 & 2.76 & 2.88 & $.85^{*}$ \\
\hline 13. Number Span (Backward) & 12 & 1.08 & 1.12 & $.53^{*}$ \\
\hline 14. Letter Span & 12 & 1.87 & 1.29 & $.67^{*}$ \\
\hline 15. Tonal Reordering & 20 & 5.82 & 3.29 & $.74^{*}$ \\
\hline 16. Letter Reordering & 20 & 9.71 & 4.26 & .62 \\
\hline 17. Rapid Spelling & 20 & 10.55 & 3.36 & .55 \\
\hline 18. Wing's Pitch Change & 20 & 6.36 & 2.26 & $.59^{*}$ \\
\hline 19. Seashore's Tonal Memory & 30 & 14.39 & 5.60 & .76 \\
\hline 20. Tonal Figures & 18 & 6.36 & 2.27 & $.50^{*}$ \\
\hline 21. Memory for Emphasis & 24 & 8.00 & 2.63 & $.49^{*}$ \\
\hline 22. Labyrinths & 24 & 11.10 & 4.37 & $.67^{*}$ \\
\hline 23. Pursuits & 48 & 42.37 & 8.10 & $.62^{*}$ \\
\hline 24. Map Planning & 20 & 6.09 & 2.70 & $.71^{*}$ \\
\hline 25. Designs & 200 & 17.15 & 8.50 & $.68^{*}$ \\
\hline 26. Hidden Figures & 15 & 9.02 & 2.85 & .56 \\
\hline 27. Copying & 32 & 18.53 & 4.85 & $.70^{*}$ \\
\hline 28. Identical Pictures & 24 & 21.89 & 3.81 & $.71^{*}$ \\
\hline 29. Name Comparison & 13 & 10.13 & 2.68 & $.70^{*}$ \\
\hline 30. Picture and Number Comparison & 24 & 14.54 & 2.74 & .68 \\
\hline 31. Intellective Masking & 20 & 15.76 & 2.45 & .44 \\
\hline 32. Compressed Speech & 70 & 6.55 & 5.51 & $.62^{*}$ \\
\hline 33. Traffic-Noise Masking & 20 & 15.15 & 2.38 & $.53^{*}$ \\
\hline 34. Seashore's Rhythm & 10 & 3.04 & 1.47 & $.57 *$ \\
\hline 35. Drake's Rhythm A & 15 & 97.50 & 29.53 & $.77^{*}$ \\
\hline 36. Drake's Rhythm B & 11 & 99.12 & 17.73 & $.79^{*}$ \\
\hline
\end{tabular}

* These are communalities from the first-order analysis.

\section{Tests}

Whenever possible, a Yugoslavian version of the test was used. Otherwise, instructions were translated from English; for some tests, the items themselves were translated as well. The list of tests used and their statistics are given in Table 2. Short descriptions of each test are as follows (asterisks indicate that the test was used by Stankov, 1971):

* Multiple-Choice Intelligibility Test. Subjects were asked to select the spoken word among the four phonetically similar words written on a sheet of paper. Yugoslavian version was created by the author. Score was number correct.

* Cloze Test. Subjects were to write down two missing words from an eight-word sentence read from the tape recorder. Yugoslavian version was created by the author. Score was number of words that would correctly complete the sentence.
* Verbal Meaning Test. This was the Yugoslavian version of the Vocabulary Test. Score was number correct.

* Social Situations Test. Subjects chose among four alternatives the one most acceptable way of behaving in typical social situations. Score was number correct.

Perception of Human Behavior Test. Yugoslavian version of a Social Intelligence Test, for example, "Old people usually claim that this world is heading to a disaster." Subjects had to indicate if the statements were likely to be true or false. Score was number correct.

Recognition of Emotions Test. Yugoslavian version of a Social Intelligence Test, for example, "I love him so much that I could drink his blood." Subjects had to choose among four emotions the one which is expressed by the statement. Score was number correct.

* Letter Series Test. Yugoslavian version (Cyrillic 
Table 3

Correlations Among Primary Factors

\begin{tabular}{|c|c|c|c|c|c|c|c|c|c|c|c|c|c|}
\hline Primary factor & 1 & 2 & 3 & 4 & 5 & 6 & 7 & 8 & 9 & 10 & 11 & 12 & 13 \\
\hline 1. Verbal Comprehension & & 69 & 54 & 37 & 46 & 39 & 37 & 40 & 53 & 37 & 62 & 52 & 29 \\
\hline 2. Experiential Evaluation & & & 31 & 28 & 31 & 21 & 23 & 20 & 32 & 36 & 49 & 29 & 18 \\
\hline 3. Induction (visual) & & & & 43 & 33 & 42 & 31 & 43 & 46 & 38 & 39 & 39 & 26 \\
\hline 4. Auditory Induction & & & & & 30 & 50 & 52 & 41 & 41 & 37 & 37 & 31 & 27 \\
\hline 5. Memory Span & & & & & & 49 & 33 & 25 & 28 & 33 & 28 & 36 & 26 \\
\hline 6. Temporal Reordering & & & & & & & 60 & 50 & 36 & 36 & 48 & 37 & 19 \\
\hline 7. Sound Pattern Recognition & & & & & & & & 45 & 33 & 22 & 37 & 37 & 29 \\
\hline 8. Relation Perception & & & & & & & & & 31 & 36 & 33 & 28 & 11 \\
\hline 9. Spatial Scanning & & & & & & & & & & 55 & 50 & 28 & 25 \\
\hline 10. Flexibility of Closure & & & & & & & & & & & 38 & 30 & 21 \\
\hline 11. Perceptual Speed & & & & & & & & & & & & 40 & 27 \\
\hline 12. Masking & & & & & & & & & & & & & 44 \\
\hline 13. Tempo & & & & & & & & & & & & & \\
\hline
\end{tabular}

Note. Decimal points are omitted.

alphabet) was created by the author. Score was number correct.

* Number Series Test. Score was number correct.

* Sequential Tonal Series Test. Four notes were played in such a way as to form a series (ascending, descending, or other). Then, three alternatives were given, and subjects were to indicate which one of them represented the continuation of the series. Score was number correct.

* Chord Series Test. Similar to the previous test.

*Tonal "Gottschaldt" Figures Test. A chord composed of three notes was presented. After this, three alternatives were given. Each alternative was a two-note interval. One of the alternatives involved two notes from the first chord. Subjects had to indicate this alternative. Score was number correct.

* Memory Span Tests (Forward and Backward Number Span and Letter Span). Tests from French et al. (1963).

* Tonal Reordering Test. Three notes were played, and after a pause, the same three notes were played again in a different order. Subjects had to write down the order in which the notes were played the second time. Score was number correct.

* Letter Reordering Test. Similar to the previous test.

* Rapid Spelling Test. Subjects had to write down words spelled at a rapid pace. Note that since Yugoslavian spelling is phonetical, the task really requires the ability to form a whole from the elements; it is not the acquired ability to spell as in English. Score was number correct.

* Wing's Pitch Change Test. Subjects had to decide whether two chords were repeated exactly or not. Score was number correct.

* Seashore's Tonal Memory Test. Pairs of tunes 3 to 10 notes long were presented. Subjects indicated which note had been changed in the second playing. Score was number correct.

* Tonal Figures Test. Subjects were given a set of four notes presented in ascending or descending order. After this, four alternatives were given. In all the alternatives, notes were played in the opposite order.
Only one alternative had the same notes as those of the first set. Subjects had to choose this alternative. Score was number correct.

* Memory for Emphasis Test. Subjects listened to a paragraph with certain words markedly emphasized. They were required to identify these words on a written script at the conclusion of the reading. Score was number correct.

Labyrinths, Pursuits, Map Planning, Designs, Hidden Figures, Copying, Identical Pictures, Name Comparison, and Picture and Number Comparison Tests. All these tests were proper markers for the primaries from French et al. (1963). They had been in use in Yugoslavia for 10 years.

* Intellective Masking Test. Subjects were asked to select from a list of phonetically similar words a word heard against an increasingly loud background of a second continuous speaker. Score was number correct.

* Compressed Speech Test. Sentences were rerecorded with an increased speed of tape movement. Subjects were to write down the sentences. Score was number of words correct.

* Traffic-Noise Masking Test. Similar to the Intellective Masking Test.

* Seashore's Rhythm Test. Subjects compared two rhythmic patterns to judge them the same or different. Score was number correct.

* Drake's Rhythm A Test. Subjects were to continue to count a beat established by a metronome during silence until told to stop. Score was number of beats different from norm.

*Drake'sRhythm B Test. Similar toDrake'sRhythm A Test, except that the interfering beat was introduced in place of the silent interval.

\section{Procedure}

All tests were recorded and presented by a tape recorder. For visual tests, the time allowed was checked by a silent interval on a moving tape. Before the start of a testing session, subjects were given answer sheets 
Table 4

Promax-Rotated (Pattern Values) Unrestricted Maximum-Likelihood Factor Analysis Solution for the Matrix of Table 3

\begin{tabular}{|c|c|c|c|c|c|}
\hline \multirow[b]{2}{*}{ Primary factor } & \multicolumn{4}{|c|}{ Factor } & \multirow[b]{2}{*}{$h^{2}$} \\
\hline & $\begin{array}{c}1 \\
(\mathrm{Gc})\end{array}$ & $\begin{array}{c}2 \\
\text { (Gf) } \\
\end{array}$ & $\begin{array}{c}3 \\
\text { (Gv?) }\end{array}$ & $\begin{array}{c}4 \\
(\mathrm{Ga} ?) \\
\end{array}$ & \\
\hline Verbal Comprehension & 1.01 & & & & 1.00 \\
\hline Experiential Evaluation & .78 & & & & .50 \\
\hline Induction (visual) & .33 & .30 & & & .39 \\
\hline Auditory Induction & & .60 & & & .43 \\
\hline Memory Span & .24 & .33 & & & .32 \\
\hline Temporal Reordering & & .95 & & & .71 \\
\hline Sound Pattern Recognition & & .81 & & & .55 \\
\hline Relation Perception & & .57 & & & .40 \\
\hline Spatial Scanning & .31 & & .32 & & .44 \\
\hline Flexibility of Closure & & & 1.02 & & 1.00 \\
\hline Perceptual Speed & .45 & .26 & & & .46 \\
\hline Masking & .32 & & & .27 & .40 \\
\hline Tempo & & & & 1.03 & 1.00 \\
\hline
\end{tabular}

Note. Gc represents the factor of Crystallized Intelligence; Gf, the factor of Fluid Intelligence; Gv, the factor of General Visualization; and $\mathrm{Ga}$, the General Auditory Function.

(and actual tests for the visual part of the battery). They were told that most of these tests had never been given to children and that the purpose of testing was to check if children could understand and do the problems. It was made clear to them that school officials would not be given the actual results of their performance. This information was sufficient to ensure good rapport.

The battery required 4 school hours, administered to each class on 2 consecutive days (2 hours each day). There were three fifth-grade and three sixth-grade classes. Special care was taken to prevent intentional or unintentional copying. During testing, two adults (not teachers) were present in the classroom.

\section{Statistical Analyses}

Two kinds of analyses were performed. First, the 20 auditory tests alone and then the full 36 -test battery were factor analyzed using the principal-components procedure. These results are not presented here because the ratio of subjects to variables was unfavorable. They served only to obtain communality estimates for those variables for which reliability estimates were not available. These communality estimates are given in Table 2.

Inspection of test means and standard deviations, relative to the total number of items, shows that the majority of tests have an adequate level of difficulty, but some appear too difficult and others too easy. Also, reliability estimates appear lower than those typically reported for standardized tests, though not considerably lower than what other investigators (e.g., Guilford, 1967) report with similar measuring instruments. To overcome these problems, $z$ scores were computed for every test and summed in sets like those in Table 1 to form composite scores for 13 primaries. The main interest of the present study was in checking the second-order structure.
The correlational matrix of composite scores, shown in Table 3, was analyzed using unrestricted maximum-likelihood factor analysis (Joreskog, 1967). ${ }^{1}$ This method was recommended by McDonald (1974) as a theoretically adequate way of avoiding the problem of indeterminacy in common factor analysis. Velicer (1976) reports that with several empirical correlational matrices, it produced solutions very similar to those of principal-components and image analyses. With this method, the obtained solution is scale free, and a statistically based test for the number of factors is available. Unrestricted maximum-likelihood factor analysis also incorporates a solution for the Heywood case (improper solution), which can occur with this method. If an improper solution obtains for some variables, say 2 out of 13 , these are expressed as principal components and partialed out of the correlation matrix $R$. The new $11 \times 11$ matrix is then analyzed using maximum likelihood, and $k-2$ factors are extracted ( $k$ being the number of factors desired). The final solution consists of combined principal-components vectors and $k-2$ vectors from the partial matrix. Communalities for variables causing the improper solution cannot be larger than one.

The number of factors was determined by a chisquare goodness-of-fit test. For the four-factor solution, a chi-square test with 35 degrees of freedom was 39.44 . This value has a probability level of .28 , indicating ac-

1 The correlational matrix of Table 3 was also analyzed by principal components, principal factors, Little Jiffy, Mark IV (Kaiser \& Rice, 1974), and Alpha-KD (Kaiser \& Case, Note 1). Unrestricted maximum. likelihood factor analysis provided a solution that closely agrees with the interpretation that could be achieved by considering all these solutions together and so was chosen here. 
ceptable fit; four factors can be used for interpretation. The matrix obtained from the unrestricted maximum-likelihood factor analysis was first rotated by varimax and then by promax (power set to 5). Table 4 contains all salient loadings (those above .20) from promax.

\section{Results}

Attending first to communalities, it can be seen that the variables with the lowest communalities are Induction (visual) and Memory Span. These are postulated measures of Gf; if they indeed measure the same factor, their loadings on it would be low. It is also apparent that three variables (Verbal Comprehension, Flexibility of Closure, and Tempo) cause an improper unrestricted maximum-likelihood factor analysis solution. In itself, this may not be reason for concern, but if these variables show high loading on one factor when other variables have low loadings, there would be strong indication that this factor is a specific one.

Inspection of Table 4 shows that the factor on which Verbal Comprehension has the highest loading (Factor 1) also has high loadings on several other factors, but the same does not hold for Tempo and Flexibility of Closure. It can be argued that these two factors should be dropped and the whole analysis should be repeated without them. This was not done with the solution of Table 4 because (a) Tempo appears to share some common variance with Masking, and Flexibility of Closure shares some variance with Spatial Scanning; (b) Tempo was a welldefined factor in the first-order analysis, and Flexibility of Closure is an established primary factor within this age group (French et al., 1963); (c) some other solutions with the matrix of Table 3 (e.g., principal components) indicate that Factors 3 and 4 might be broader than those produced by unrestricted maximum-likelihood factor analysis.

Although Tempo and Flexibility of Closure were retained, it is important to observe caution in interpreting Factors 3 and 4, since clearly, they are not as broad as one would hope. The following discussion assumes that the data provide evidence for Factors 1 and 2 and that the other two factors are implied but not definitely established.

\section{Factor 1: Crystallized Intelligence $(G c)$}

Two variables with high loadings on this factor are Verbal Comprehension and Experiential Evaluation. They are considered to be good markers of crystallized intelligence, since both of them imply the effect of acculturation. Several other variables also have noteworthy loadings on this factor, the highest being Perceptual Speed. This primary is typically found to be a good marker for Gv (and also Broad Speediness, or Gs), and it is necessary to explain its appearance here. Three tests were used to define this primary: Identical Pictures, Number and Picture Comparison (a subtest from the Beta test), and Name Comparison. The last-mentioned test involved comparison of long Yugoslavian names, and it can be considered to be a measure of learning and acculturation in this age group. In other words, it can be claimed that spelling has not been mastered within this age group; therefore, this test does not measure pure Perceptual Speed and should be an indicant of $\mathrm{Gc}$ as well. Also, the Perceptual Speed loading may reflect the fact that part of $\mathrm{Gc}$ is measured by visual tests.

We should keep in mind that Gf-Gc theory accepts the possibility of overlap among the factors and allows that one variable can measure two (or more) factors. This would then explain Gc loadings on other variables as well. For example, Spatial Scanning also loads this factor, indicating again that part of $\mathrm{Gc}$ is measured through the visual modality. Masking would implicate auditory input too, and finally, Memory Span and Induction point to a well-known overlap between Gf and Gc. Note, however, that all the other variables besides Verbal Comprehension and Experiential Evaluation do not have high loadings on this factor, and therefore, it can be concluded that this is a broad Gc factor.

Factor 2: Fluid Intelligence (Gf) Measured Through Auditory Modality

Temporal Reordering, Sound Pattern Recognition, Auditory Induction, and Relation Perception show high loadings on this factor. Somewhat lower, but still 
noteworthy, are loadings of Memory Span, Induction, and Perceptual Speed. This is obviously a rather broad factor and its interpretation is not easy. Most primaries of this factor, and in particular those with high loadings, are new auditory primaries that have been insufficiently explored. There are several possibilities.

It may be argued that since music is taught in schools and some of the tests involve tones, this is another Gc factor reflecting specific acculturation influence. That would be an unlikely conclusion because not all primaries of this factor involve musical stimuli, and even for those that do, it is hard to see why performance on them would depend on musical training.

Another possible hypothesis may be that this is a General Auditory Function (Ga). This is probably not the case because all the primaries involved require relatively complex manipulation of stimulus input, not merely perception. Also, only one of the two "pure" markers for $\mathrm{Ga}$ (Masking) has a low salient loading. In addition, it can be seen that two primaries (Perceptual Speed and Induction) are visual. While visual and auditory processes may correlate, such correlation is likely to be lower between the perceptual than between "higher" mental processes (Stankov, 1971). In other words, it is more probable that a factor defined by both auditory and visual primaries represents higher processes than those typically implied by the word perception and by Ga.

Among the other possibilities that should be considered are (a) short-term acquisition and retrieval function (SAR), (b) intermediate memory, and (c) fluid intelligence (Gf). All these are more likely candidates than either $\mathrm{Ga}$ or $\mathrm{Gc}$; indeed, in the earlier writings of Horn, they were all treated as one broad factor, that of fluid intelligence. SAR, which is to some extent similar to Jensen's Level I ability, is measured by tests of immediate and associative memory. Since Memory Span has a relatively low loading on this factor and no primary represents a clear measure of associative memory, it would be inappropriate to identify it as such, but a certain similarity should be acknowledged. Recently, Horn (1975) indicated that in the life-span development of intelligence, another broad factor corresponding to the process of intermediate memory (memory for events lasting more than $30 \mathrm{sec}$ but less than 1 hour) can be detected. He quotes some of the tests and primaries of Factor 2 as appropriate measures of it. On the other hand, there is as yet little evidence showing clearly the distinction between intermediate memory and other broad factors.

Working with adults, Stankov (1971) found that a majority of tests used to measure primaries of the present Factor 2 define a second-order factor identified as Gf. It is true that the highest loadings in the 1971 study were those of Memory-Span tests, and possibly a reinterpretation of these data along the lines of a new SAR factor should be attempted. Also, while the loading of Induction on Factor 2 is relatively low, its presence along with Memory Span suggests, at least in part, the processes called fluid intelligence. Factor 2 is therefore tentatively called fluid intelligence measured through auditory modality, with the understanding that it also embraces processes of SAR and intermediate memory.

\section{Factor 3: General Visualization $(G v)$ ?}

As already noted, the last two factors are rather narrow: Only two primaries have salient loadings on them. Factor 3 is predominantly Flexibility of Closure with a much lower loading of Spatial Scanning. While Perceptual Speed loads Gc and Gf, it is worth noting that in one of the other solutions attempted with this correlational matrix (Alpha-KD; Kaiser \& Case, Note 1), it did load on this factor.

Undheim (1976) reported a broad Visualization factor among the 10 to 11 year olds in Norway, and present results are obviously in poor agreement with his. But Undheim's broad Visualization factor might not be broader than the one we have here. He had postulated three visualization primaries: (a) Spatial Relation (S), measured by two tests that produced one factor at the first order; (b) Speed of Closure (Cs), measured by parallel forms of the same test (producing a doublet at the first order); and (c) Visualization primary $\left(V_{z}\right)$, measured by three 
Table 5

Factor Intercorrelations

\begin{tabular}{crrrr} 
& \multicolumn{5}{c}{ Factor } \\
\cline { 2 - 5 } Factor & Gc & \multicolumn{1}{c}{ Gf } & Gv & Ga \\
\hline Gc & 1.00 & .63 & .51 & .40 \\
$\mathrm{Gf}$ & .63 & 1.00 & .50 & .40 \\
$\mathrm{Gv}$ & .51 & .50 & 1.00 & .25 \\
$\mathrm{Ga}$ & .40 & .40 & .25 & 1.00 \\
\hline
\end{tabular}

Note. Gc represents the factor of Crystallized Intelligence; Gf, the factor of Fluid Intelligence; $\mathrm{Gv}$, the factor of General Visualization; and $\mathrm{Ga}$, the General Auditory Function.

tests that did not load the same factor at the first order. Because of this outcome at the first order, he included S and Cs composites and all three $\mathrm{Vz}$ tests in his analysis at the second order. His low loading (.22) for $\mathrm{Cs}$ on General Visualization is analogous here to what happened with Perceptual Speed. The highest loading on $\mathrm{Gv}$ was .60 for Spatial Relations, whereas all three Visualization tests had loadings in the $.40 \mathrm{~s}$. If the three Visualization tests had been combined into one $\mathrm{Vz}$ composite, his result might look similar to the present solution. The breadth of his $\mathrm{Gv}$ factor depends on the inclusion of separate $\mathrm{Vz}$ tests in the second-order analysis.

\section{Factor 4: General Auditory Function $(G a)$ ?}

Like Gv, this factor is mainly Tempo with a low loading of Masking. Ga and Gv show the lowest correlation among all factor intercorrelations of Table 5 . This agrees with the expectation that perceptual factors should have low correlations among themselves.

\section{Discussion}

In interpreting results, one should keep in mind that instructions for all tests, and some tests themselves, were given in a different language from that in which they were standardized, and some tests might be poorly adapted for this age group.

The results, nonetheless, indicate two of the four hypothesized factors. One of these represents crystallized intelligence; the other was tentatively labeled as fluid intelligence measured with auditory tests. The two perceptual factors are less well established, so their occurrence here can be viewed with suspicion. Such an outcome might occur if some factors were not sufficiently overdetermined. With primaries based on composites derived to a larger extent from the first-order analysis, better overdetermination could have been achieved. Even with this reservation, the present results deserve consideration with respect to the age-differentation hypothesis.

With this age group, one broad factor should reflect acculturational influences (especially in a school system that allows little specialization at this age level). A broad Gc factor did emerge here. It was also hypothesized that if Gf appeared in addition to $\mathrm{Gc}$, their correlation should be higher than what is typically obtained with adults (between .20 and .50). As seen in Table 5, this correlation (.63) is close to Undheim's (1976) result of .64 . He also obtained comparable correlations between $\mathrm{Gv}, \mathrm{Gc}$, and Gf. On the other hand, his fourth factor (broad Speediness) had higher correlations with the first three factors than $\mathrm{Ga}$ had in the present study.

Some differentiation of abilities seems to have taken place, and although a clear $\mathrm{Gc}$ factor did occur, some additional common variance also exists. The reason for separation between $\mathrm{Gf}$ andGc was attributed by Undheim to motivational and other noncognitive factors in addition to age, but neither the present data nor Undheim's provide a direct check of this.

A broad $\mathrm{Gv}$ factor has commonly been observed among adults, in addition to Gf and Gc. Comalli (1970) reviewed data on visual illusions, spatial orientation, part-whole differentiation, perceptual closure, and speed of recognition. Most of these variables show progressive changes during childhood until, at the ages between 14 and 20 years, performance is typically similar to that of adults. Since development of $\mathrm{Gv}$ must be related to these changes, it is not surprising to find that at the age of 11 to 12 years, its showing appears poor and only implied.

General Auditory Function, as the studies with adults show, correlates with audiometer measures, a finding that implies deteriora- 
tion of hearing acuity. Since this should not be a factor during childhood years on tests using typical speech sounds (e.g., Masking), perhaps a well-established $\mathrm{Ga}$ factor should not be expected. The present results indicate also that some individual differences occur with respect to Tempo (Rhythm), which while not directly related to hearing acuity has been found to measure $\mathrm{Ga}$ in adults.

General Auditory Function may be poorly defined here because the auditory domain has not been sufficiently explored; the auditory primaries are then less well established than the visual ones. The inclusion of other primaries might define $\mathrm{Ga}$ more firmly.

Another possibility is that in this study, the primaries themselves have not been replicated. The assumption that the primaries here are the same as in Stankov (1971) may not be justified. The first-order analysis, although performed, has not been reported here. It showed, however, that Tempo, Auditory Induction, Temporal Reordering, and Masking do appear at the first order.

The fact that Gf and Gc have been replicated with auditory tests is of considerable importance of temporal integration as part two board factors. Stankov (1971) elaborated on several characteristics of auditory tests that bring into focus some features of intellectual functioning not easily accessed by ordinary visual tests. For example, sequential presentation of stimuli, which is typical of auditory material, stresses the importance of temporal integration as part of intelligence. The paced and controlled nature of presentation as compared to relatively free work through the visual tests may cast a new light on the role of intellectual speed. This and also the uncertainty associated with the need to keep in mind several stimuli spread over time may contribute to our understanding of the role of attentiveness and of the relationship with other personality variables such as risk taking. On the practical side, measurements of Gf and $\mathrm{Gc}$ through the auditory modality opens up the possibility of assessing the capabilities of various disadvantaged groups (e.g., the blind).
The two perceptual factors ( $\mathrm{Gv}$ and $\mathrm{Ga}$ ) represent cognitive processes that seem relatively less complex than those required by intelligence tests. They are dependent, according to the theory, on the functioning of peripheral organs, afferent pathways, and projection areas of the visual and auditory cortex. Bock (1973) indicates that the left cerebral hemisphere may be responsible for the processing of $\mathrm{Gc}$ and some aspects of $\mathrm{Ga}$. Both $\mathrm{Ga}$ and $\mathrm{Gc}$ seem to be restricted somewhat (possibly.artificially) in the scope of material used to assess them. Gv seems to emphasize pictorial material (excluding color and other dimensions of visual perception); Ga seems to be restricted to spoken words, time perception, and tones varying in pitch (excluding loudness, space localization, etc.). Finally, Ga appears to be closely linked to measures of auditory acuity, which seems not to be the case for Gv.

\section{Reference Note}

1. Kaiser, H. F., \& Case, D. Computer algorithm for Alpha-KD (mimeographed paper). Berkeley: University of California, Department of Education, 1975.

\section{References}

Bock, D. R. Word and image: Source of the verbal and spatial factors in metal test scores. Psychometrika, 1973, 38, 437-458.

Cattell, R. B. Theory of fluid and crystallized intelligence: A critical experiment. Journal of Educational Psychology, 1963, 54, 1-22.

Cattell, R. B. The theory of fluid and crystallized general intelligence checked at the 5-6 year old level. British Journal of Educational Psychology, 1967, 37, 209-224.

Cattell, R. B. Abilities: Their structure, growth, and action. Boston: Houghton Mifflin, 1971.

Comalli, P. E. Life-span changes in visual perception. In L. R. Goulet \& P. B. Baltes (Eds.), Life-span developmental psychology: Theory and research. New York: Academic Press, 1970.

French, J. W., Ekstrom, R. B., \& Price, L. A. Manual for Kit of Reference Tests for Cognitive Factors. Princeton, N.J.: Educational Testing Service, 1963.

Guilford, J. P. The nature of human intelligence. New York: McGraw-Hill, 1967.

Horn, J. L. Organization of abilities and the development of intelligence. Psychological Review, 1968, $75,242-259$.

Horn, J. L. Organization of data on life-span development of human abilities. In R. Goulet \& P. B. 
Baltes (Eds.), Life-span developmental psychology: Theory and research. New York: Academic Press, 1970.

Horn, J. L. Theory of functions represented among auditory and visual test performances. In J. R. Royce (Ed.), Contributions of multivariate analysis to psychological theory. New York: Academic Press, 1974.

Horn, J. L. Psychometric studies of aging and intelligence. In S. Gershon \& A. Raskin (Eds.), Geriatric psychopharmacology: The scene today. New York: Raven Press, 1975.

Horn, J. L., \& Cattell, R. B. Age differences in fluid and crystallized intelligence. Acta Psychologia, 1967, 26, $107-119$.

Joreskog, K. G. Some contributions to maximum likelihood factor analysis. Psychometrika, 1967, 32, 443-482.

Kaiser, H. F., \& Rice, P. Little Jiffy, Mark IV. Edu- cational and Psychological Measurement, 1974, 34, 111-117.

McDonald, R. P. The measurement of factor indeterminancy. Psychometrika, 1974, 39, 203-222.

Stankov, L. Hierarchical structure of auditory abilities and relationship between auditory and visual modalities. Unpublished doctoral dissertation, University of Denver, 1971.

Undheim, J. O. Ability structure in 10-11-year-old children and the theory of fluid and crystallized intelligence. Journal of Educational Psychology, 1976, 68, 411-423.

Velicer, W. F. An empirical comparison of the similarity of principal component, image, and maximum likelihood factor patterns. Multivariate Behavioral Research, 1976, 12, 3-23.

Received November 4, 1976 